\title{
How diet interacts with longevity genes
}

\author{
Andrzej Bartke, Michael Bonkowski, Michal Masternak
}

Departments of Internal Medicine, Physiology and Pharmacology, Southern Illinois University School of Medicine, Springfield, IL, USA

\begin{abstract}
In laboratory mice, suppression of growth hormone (GH) signaling by spontaneous mutations or targeted disruption of GH- or IGF1-related genes can lead to an impressive increase of longevity. Hypopituitary Ames dwarf (Prop1 ${ }^{\text {df }}$ ) and GH receptor knockout (GHRKO) mice live 35-70\% longer than their normal littermates. Many phenotypic characteristics of these longlived mutants resemble findings in genetically normal animals subjected to calorie restriction (CR). Microarray and RT-PCR studies of gene expression suggest that effects of the "longevity assurance genes" (Prop1 ${ }^{\text {df }}$ or Ghr-/-) and CR are overlapping but not identical. Subjecting Ames dwarf mice to $30 \%$ CR starting at 2 months of age leads to a further significant extension of their average and maximal lifespans. In contrast, identical $C R$ regimen has either no or very little effect (depending on gender) on longevity of GHRKO mice. We suspect that this difference in response is related to the fact that $\mathrm{CR}$ improves insulin sensitivity in Ames dwarfs but does not further increase the extreme insulin sensitivity of GHRKO mice. To search for effects of CR associated with extension of longevity, we are studying expression of insulin and IGF1-related genes in the liver, skeletal muscle and heart of normal and GHRKO mice. Results obtained to date suggest that reduced Akt phosphorylation and PPAR $\beta / \delta$ expression in the liver, reduced JNK1 phosphorylation and increased PGC1 $\alpha$ expression in the muscle, and increased expression of IGF1 and insulin receptor in the heart are either related to mechanisms of CR action on longevity or represent potential biomarkers of delayed aging.
\end{abstract}

Key words: Aging, Ames dwarf, Calorie restriction, Growth hormone receptor knockout, Growth hormone deficiency, Insulin sensitivity, Lifespan, Longevity

\section{INTRODUCTION}

Aging and lifespan, like other characteristics of living organisms, are determined by a complex inter-

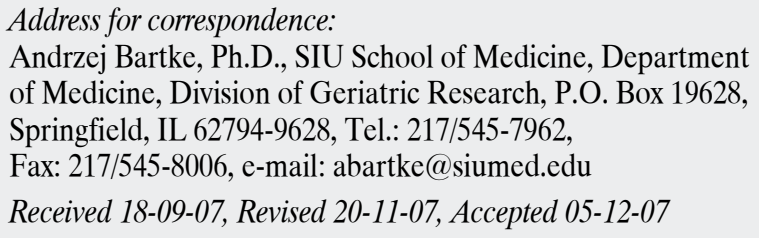

play of genes and environment. Nutrient availability is particularly important among the environmental factors affecting aging. While adequate sources of energy and essential nutrients are absolute requirements for normal growth and reproductive competence, unlimited access to high quality food is not optimal for longevity. The negative impact of gluttony on hu- 
man health was suggested in medical writings dating back to the Renaissance, ${ }^{1}$ and studies conducted in the 1930s provided clear evidence that domesticated strains of rats and mice maintained in the laboratory environment will live significantly longer if their food intake is reduced below the amount they would ordinarily consume. ${ }^{2,3}$ This intervention, termed "calorie restriction" (CR) or "dietary restriction," reliably increases median, average and maximal lifespan in laboratory rodents and exerts similar effects in other species, almost certainly including non-human primates and humans. ${ }^{4-7}$ Importantly, lifespan extension produced by CR is associated with delayed onset, reduced incidence and reduced severity of cancer and other age-associated diseases and with preservation of a "youthful" phenotype.

Studies suggesting that mutations of a single gene can profoundly affect lifespan of mice date back to the early '70s. ${ }^{89}$ Work conducted during the past 15 years has provided firm evidence that mutations causing deficiency of several anterior pituitary hormones, isolated growth hormone $(\mathrm{GH})$ deficiency or GH resistance increase lifespan of mice. $(10-12$; reviewed in 13). The extension of longevity in these mutants was comparable to the effects of reducing calorie intake in genetically normal animals by 30 $50 \%$ and was also associated with delayed onset and reduced incidence of age-associated disease and with multiple symptoms of delayed aging, closely resembling the findings in CR animals. However, normal (voluntary) food intake of these long-lived mutants was not reduced and, in contrast to the effects of CR, their adiposity was generally increased, in some cases quite drastically. Therefore, it became of interest to compare and contrast the physiological characteristics of long-lived mutants and genetically normal ("wild-type") animals subjected to CR and to examine the interaction of $\mathrm{CR}$ with "longevity assurance genes".

In this chapter, we will briefly describe the origin and key characteristics of two long-lived mouse mutants, compare the effects of CR in these mutants and in normal animals and conclude with highlights of our recent and ongoing studies aimed at identifying the mechanisms responsible for the impact of $\mathrm{CR}$ and reduced $\mathrm{GH}$ signaling on longevity.

\section{AMES DWARF MICE}

Ames dwarf mice are homozygous for a spontaneous mutation that was discovered in a research mouse colony at Iowa State University. The animals were first described by Schaible and Gowen in $1961^{14}$ and subsequently shown to be GH, prolactin (PRL) and thyrotropin (TSH) deficient. $(15,16$; reviewed in 17$)$ Sornson et $\mathrm{l}^{18}$ have shown that Ames dwarfism was due to a loss-of-function mutation of a gene responsible for an early stage of differentiation of anterior pituitary cells destined to become somatotrops, lectotrops and thyrotrops and named this gene Prophet of pituitary factor 1, (Pit1) or "Prop1." Ames dwarf mice appear normal at birth but grow slowly and reach less than one half of the adult body weight of their normal siblings. Puberty is markedly delayed and females are sterile due to PRL deficiency. ${ }^{16,17}$ Consistent with GH and TSH deficiency, adult Ames dwarf mice have extremely low levels of circulating insulin-like growth factor1 (IGF1) and thyroid hormones. ${ }^{17}$ They have reduced body temperature, ${ }^{19}$ but food consumption and oxygen utilization per gram of body weight are increased. ${ }^{20,21}$ Circulating glucose and insulin levels are reduced under both fasted and fed conditions, implying that increased insulin sensitivity and blood glucose suppression during insulin tolerance test is indeed significantly greater in Ames dwarfs than in normal mice. ${ }^{22,23}$ Adiposity of young adults is increased, which could be expected from GH deficiency and hypothyroidism, but circulating levels of adiponectin are increased, ${ }^{24}$ and age-related increase in percent body fat is less pronounced than in normal animals of the same strain. ${ }^{25}$ Median, average and maximal lifespan are significantly increased in both sexes of Ames dwarf mice, with the increase in average lifespan ranging from approximately $35 \%$ to as much as $70 \%$, depending on sex and diet. ${ }^{10,17,26}$ Comparable extension of lifespan along with evidence for delayed aging of the immune system, joint cartilage and collagen properties were described in Snell dwarf mice, animals that have identical primary hormonal defects due to mutation of the Pit1 gene, located on a different chromosome than Prop1.,11 Little (Ghrhrit) mice with isolated GH deficiency exhibit a smaller but statistically significant increase in longevity. ${ }^{11}$

Available evidence suggests that the remarkable 
increase in longevity of Ames dwarf mice is due to multiple mechanisms, including reduced IGF1 and mammalian Target Of Rapamycin (mTOR) signaling, ${ }^{27,28}$ delayed onset and reduced incidence of fatal neoplastic disease, ${ }^{29}$ increased sensitivity to insulin combined with reduced insulin levels, ${ }^{22,23}$ hypothyroidism and reduced body core temperature,${ }^{19}$ increased activity of antioxidant enzymes and reduced oxidative damage $(30,31$; reviewed in 17$)$ and resistance to various forms of oxidative, toxic and metabolic stress. ${ }^{32,33}$

\section{GROWTH HORMONE RECEPTOR KNOCKOUT (GHRKO) MICE}

Mice with deficiency of GH receptor and consequent $\mathrm{GH}$ resistance were produced in the laboratory of John Kopchick by disruption of the Ghr gene. ${ }^{34}$ Circulating IGF1 levels in GHRKO mice are very low, ${ }^{34}$ while IGF expression in different organs is variably reduced or normal ${ }^{35}$ and plasma $\mathrm{GH}$ levels are elevated, reflecting a lack of negative GH/IGF1 feedback. ${ }^{34}$ GHRKO (Ghr -/-) animals are small with nearly normal body proportions. Both sexes are fertile but puberty is delayed, and many measures of reproductive competence and testicular endocrine function are quantitatively reduced. ${ }^{34,36-39}$ Insulin levels are drastically reduced, glucose levels are "low normal" or slightly reduced and insulin sensitivity is increased. ${ }^{34,40}$ Plasma adiponectin levels are elevated in spite of significantly increased adiposity. ${ }^{41}$

Median, average and maximal lifespan are increased in both sexes. ${ }^{12,43,44}$ Extended longevity of GHRKO mice was observed in two different labs, across three different genetic backgrounds and using diets differing with regard to the source of protein and the content of phytoestrogens. ${ }^{12,26,42-44}$ Putative mechanisms of delayed aging and increased longevity of GHRKO mice resemble those listed above for the Ames dwarfs. (32, 34, 40, 41, 44, Ikeno and Bartke, unpublished; reviewed in 13, 17).

\section{EFFECTS OF CALORIE RESTRICTION IN AMES DWARF AND GHRKO MICE}

Starting at approximately 2 months of age, Ames dwarfs, GHRKO mice and normal siblings of these mutants were subjected to $30 \% \mathrm{CR}$, introduced gradually, over a period of 2 weeks. ${ }^{20}$ The CR animals were fed daily an amount of food corresponding to $70 \%$ of the amount consumed by animals of the same genotype, age and sex, which had unlimited access to food. When the consumption of food by the ad libitum (AL) animals started a decline that is the expected correlate of aging, the amount of food given daily to the CR mice was not reduced in parallel but remained constant for the remainder of their lifespan. In normal mice, $\mathrm{CR}$ produced the expected increase in longevity. Lifespan of long-lived Ames dwarf mice was further extended by $30 \% \mathrm{CR}$. This included increases in median, average and maximal lifespan that were significant in both sexes. ${ }^{44}$ In contrast to its effects in Ames dwarfs, CR had very little effect on the longevity of GHRKO mice: median and average longevity were not affected in either sex, while maximal lifespan (calculated as average lifespan of the oldest $10 \%$ of the animals) was increased only in females. ${ }^{45}$

The difference between the effects of identical regimens of CR on longevity of Ames dwarf and GHRKO mice was unexpected in view of considerable similarities of phenotypic characteristics of these mutants and presumably comparable mechanisms of their extended longevity. These differences provide an opportunity to separate those effects of CR that are associated with an increase in lifespan from those that are not. Highlights of studies aimed at identifying the mechanisms that could link specific effects of $\mathrm{CR}$ with increased lifespan are described in the next section of this article. Explaining why GHRKO mice derived little (females) or no (males) longevity benefits from being subjected to CR should be particularly informative, because failure of $\mathrm{CR}$ to affect median and average lifespan in either sex is very rare if not unprecedented. Various regimens of CR resulted in extended longevity in hundreds of experiments involving different strains of mice and rats, as well as unrelated species including taxonomically distant animals and unicellular yeast.

\section{SEARCH FOR MECHANISMS OF ACTION OF CR IN LONG-LIVED MUTANT MICE}

As mentioned earlier, both Ames dwarf and GH- 
RKO mice have reduced levels of insulin, reduced or "low normal" levels of glucose and enhanced sensitivity to exogenons insulin. ${ }^{22,23,34,40}$ On the basis of these observations and the documented relationships between aging, insulin resistance and age-related disease, we have proposed that enhanced insulin sensitivity is a crucial component in mediating the effects of reduced somatotropic signaling on aging and longevity. ${ }^{13,17,23}$ It is well documented that CR reduces circulating levels of insulin and improves insulin sensitivity. ${ }^{3}$ In our studies, $30 \%$ CR increased insulin sensitivity in normal siblings of Ames dwarf and GHRKO mice, caused further significant increase in the insulin sensitivity of Ames dwarfs, but did not alter the (already high) insulin sensitivity of GHRKO mice (45, Masternak, unpublished). These observations support the suggested role of enhanced insulin sensitivity in the extended longevity of these mutants and imply that the failure of CR to increase lifespan of GHRKO mice may have been due to the inability of this intervention to produce further enhancement of insulin sensitivity in these hypoinsulinemic and remarkably insulin-sensitive animals.

We are currently examining acute effects of intravenous insulin administration on phosphorylation of insulin receptor and insulin receptor substrates in the liver and muscle of normal and GHRKO mice fed AL or subjected to CR to elucidate the mechanisms of altered whole-animal insulin sensitivity. To further analyze this and other potential mechanisms of differential responses of normal, Ames dwarf and GHRKO mice to CR, we have analyzed gene expression with emphasis on genes related to IGF1 and insulin signaling.

Microarray analysis of hepatic gene expression was conducted in collaboration with Drs. Richard Miller and Steven Spindler and led to the demonstration that effects of these life-extending mutations on gene expression were distinctly different from the effects of CR, although some overlap was detected. ${ }^{46,47}$ Interestingly, effects of CR on the expression of those genes that were influenced by CR in both normal and GHRKO mice were proportionally greater in normal animals. ${ }^{46}$ Studies of the expression of individual genes in the liver, skeletal muscle, heart and adipose tissue by real-time PCR and measurements of the corresponding protein products and their phospho- rylation revealed a number of differences between the responses of normal and GHRKO mice to CR. Activation of protein kinase $\mathrm{B}(\mathrm{Akt})$ is important in mediating the actions of both IGF1 and insulin, and studies in Akt2-KO animals revealed a major role of Akt in mediating the effects of CR on glucose uptake in the muscle. ${ }^{48}$ The levels of Akt2 in the muscle of GHRKO mice were increased in comparison to the normal animals in association with enhanced insulin sensitivity of these animals. ${ }^{49}$ Calorie restriction increased muscle Akt2 levels in normal but not in GHRKO animals, ${ }^{49}$ corresponding to CR effects on responses to exogenous insulin in the insulin tolerance test and its effects on longevity. ${ }^{45}$

C-jun N-terminal kinase (JNK) inhibits insulin signaling by increasing serine phosphorylation of IRS$1 .^{50}$ The levels of active (phosphorylated) JNK-1 were reduced in the muscle of GHRKOs, as compared to normal mice, presumably contributing to enhanced insulin sensitivity by reducing the inhibitory influence of JNK. In response to CR, the levels of active JNK-1 were reduced in normal, but not affected in GHRKO animals, ${ }^{49}$ again corresponding to the effects of CR on insulin sensitivity and longevity.

It was recently reported that insulin sensitivity is enhanced and lifespan is increased in mice heterozygous for the IRS-2 deletion. ${ }^{51}$ Unexpectedly, hepatic expression of IRS-2 was increased in both Ames dwarf and GHRKO in comparison to normal animals from the same strains. ${ }^{52,53}$ Subjecting GHRKO mice to CR did not alter hepatic IRS-2 expression and reduced IRS-2 mRNA levels in the skeletal muscle. ${ }^{53}$ It is difficult to delineate the interactive effects of CR and longevity genes on lifespan and whole-animal insulin sensitivity from their effects on expression of insulin- and IGF1-related genes, because these effects are different in different organs. For example, in GHRKO mice, the levels of PPAR $\gamma$ are increased in the liver, reduced in the muscle and not altered in the heart. ${ }^{54,55}$ The levels of PPAR $\alpha$ protein are increased in the liver but reduced in the muscle and heart..$^{54,55}$

Ongoing studies in our laboratory indicate that the impact of CR on phosphorylation of insulin receptor and IRS-1 in response to acute insulin stimulation in vivo can differ substantially between different insulin target organs (Bonkowski, Dominici and 
Bartke, unpublished). For example, failure of CR to enhance whole-animal insulin sensitivity in GHRKO mice appears to be unrelated to the alterations in the levels and tyrosine phosphorylation of hepatic insulin receptor but closely correlates with lack of changes in serine phosphorylation of IRS-1 in the muscle.

Results of studies of the interaction of two different longevity assurance genes with $\mathrm{CR}$ in mice can be summarized as follows:

i. Mutations which cause $\mathrm{GH}$ deficiency or resistance produce phenotypic changes that resemble but do not mimic the effects of CR in genetically normal animals, including delayed aging and increased lifespan.

ii. Identical regimens of CR produce further extension of longevity in hypopituitary, GH-deficient Ames dwarf mice but not in GH-resistant GHRKO mice.

iii. Effects of longevity genes and CR, singly or combined, on lifespan are associated with the corresponding changes in whole-animal insulin sensitivity. These associations add to the evidence that altered insulin signaling is an important mediator of the effects of altered somatotropic signaling on longevity.

iv. Effects of reduced GH signaling and CR on insulin sensitivity reflect complex, organ-specific alterations in the expression of insulin-related genes, the levels of their protein products and phosphorylation of these proteins.

\section{Acknowledgements}

These studies were supported by the NIH through grants AG 19899 and U19 AG023122, and by the Ellison Medical Foundation. Contributions of many colleagues and former students and especially Drs. F. Dominici, D. Turyn, K. Al-Regaiey and Z. Wang are gratefully acknowledged.

\section{REFERENCES}

1. Cornaro L 1917 The Art of Living Long. Milwaukee: William F. Butler.

2. McCay CM, Crowell MF, Maynard LA, 1989 The effect of retarded growth upon the length of the life span and upon the ultimate body size. 1935. Nutrition 5: 155-171.

3. Weindruch R, Walford RL 1988 The retardation of aging and disease by dietary restriction. Springfield, IL: Charles C. Thomas.

4. Bodkin N, Alexander T, Ortmeyer H, Johnson E, Hansen B, 2003 Mortality and morbidity in laboratory-maintained Rhesus monkeys and effects of long-term dietary restriction. J Gerontol A Biol Sci Med Sci 58: 212-219.

5. Messaoudi I, Warner J, Fischer M, et al, 2006 Delay of $\mathrm{T}$ cell senescence by caloric restriction in aged longlived nonhuman primates. Proc Natl Acad Sci USA 103: 19448-19453.

6. Willcox DC, Willcox BJ, Todoriki H, Curb JD, Suzuki M, 2006 Caloric restriction and human longevity: what can we learn from the Okinawans? Biogerontology 7: 173-177.

7. Holloszy JO, Fontana L, 2007 Caloric restriction in humans. Exp Gerontol 42: 709-712.

8. Silberberg R, 1972 Articular aging and osteoarthrosis in dwarf mice. Pathol Microbiol 38: 417-430.

9. Fabris N, Pierpaoli W, Sorkin E, 1972 Lymphocytes, hormones, and ageing. Nature 240: 557-559.

10. Brown-Borg HM, Borg KE, Meliska CJ, Bartke A, 1996 Dwarf mice and the ageing process. Nature 384: 33.

11. Flurkey K, Papaconstantinou J, Miller RA, Harrison DE, 2001 Lifespan extension and delayed immune and collagen aging in mutant mice with defects in growth hormone production. Proc Natl Acad Sci USA 98: 6736-6741.

12. Coschigano KT, Holland AN, Riders ME, et al, 2003 Deletion, but not antagonism, of the mouse growth hormone receptor results in severely decreased body weights, insulin, and insulin-like growth factor I levels and increased life span. Endocrinology 144: 3799-3810.

13. Bartke A 2006 Life extension in the dwarf mouse. In: Conn PM (ed) Handbook of models for the study of human aging, Elsevier Academic Press; pp, 403-414.

14. Schaible R, Gowen JW, 1961 A new dwarf mouse. Genetics 46: 896 .

15. Bartke A, 1964 Histology of the anterior hypophysis, thyroid and gonads of two types of dwarf mice. Anat Rec 149: 225-235.

16. Bartke A, 1965 Influence of luteotrophin on fertility of dwarf mice. J Reprod Fert 10: 93-103.

17. Bartke A, Brown-Borg H, 2004 Life extension in the dwarf mouse. Cur Top Dev Biol 63: 189-225.

18. Sornson MW, Wu W, Dasen JS, et al, 1996 Pituitary lineage determination by the prophet of pit-1 homeodomain factor defective in Ames dwarfism. Nature 384: 327-333.

19. Hunter WS, Croson WB, Bartke A, Gentry MV, Meliska CJ, 1999 Low body temperature in long-lived Ames dwarf mice at rest and during stress. Physiol Behav 67: 433-437.

20. Mattison JA, Wright JC, Bronson RT, et al, 2000 Studies of aging in Ames dwarf mice: Effects of caloric restriction. J Am Aging Assoc 23: 9-16.

21. Westbrook R, Bonkowski MS, Strader AD, Bartke A 2007 Oxygen consumption, respiratory quotient, and heat production in long-lived and Ames dwarf mice, and 
short-lived BGH Transgenic mice. In: American Aging Association 36th Annual Meeting, June 1-4, 2007; San Antonio, Texas.

22. Borg KE, Brown-Borg HM, Bartke A, 1995 Assessment of the primary adrenal cortical and pancreatic hormone basal levels in relation to plasma glucose and age in the unstressed Ames dwarf mouse. Proc Soc Exp Bio Med 210: 126-133.

23. Dominici FP, Hauck S, Argentino DP, Bartke A, Turyn D, 2002 Increased insulin sensitivity and upregulation of insulin receptor, insulin receptor substrate (IRS)-I and IRS-2 in liver of Ames dwarf mice. J Endocrinol 173: 81-94.

24. Wang Z, Masternak MM, Al-Regaiey KA, Bartke A, 2007 Adipocytokines and the regulation of lipid metabolism in growth hormone transgenic and calorie-restricted mice. Endocrinology 148: 2845-2853.

25. Heiman M, Tinsley F, Mattison J, Hauck S, Bartke A, 2003 Body composition of prolactin-, growth hormone-, and thyrotropin-deficient Ames dwarf mice. Endocrine 20: 149-154.

26. Bartke A, Peluso MR, Moretz N, et al, 2004 Effects of soy-derived diets on plasma and liver lipids, glucose tolerance, and longevity in normal, long-lived and short-lived mice. Horm Metab Res 36: 550-558.

27. Chandrashekar V, Bartke A, 1993 Induction of endogenous insulin-like growth factor-I secretion alters the hypothalamic-pituitary-testicular function in growth hormone-deficient adult dwarf mice. Biol Reprod 48: 544-551.

28. Sharp ZD, Bartke A, 2005 Evidence for down-regulation of phosphoinositide 3-kinase/Akt/mammalian target of rapamycin (PI3K/Akt/mTOR)-dependent translation regulatory signaling pathways in Ames dwarf mice. $\mathbf{J}$ Gerontol A Biol Sci Med Sci 60: 293-300.

29. Ikeno Y, Bronson RT, Hubbard GB, Lee S, Bartke A, 2003 Delayed occurrence of fatal neoplastic diseases in Ames dwarf mice: correlation to extended longevity. J Gerontol A Biol Sci Med Sci 58: 291-296.

30. Romanick MA, Rakoczy SG, Brown-Borg HM, 2004 Long-lived Ames dwarf mouse exhibits increased antioxidant defense in skeletal muscle. Mech Ageing Dev 125: 269-281.

31. Brown-Borg HM, Rakoczy SG, 2005 Glutathione metabolism in long-living Ames dwarf mice. Exp Gerontol 40: $115-120$.

32. Harper JM, Durkee SJ, Dysko RC, Austad SN, Miller RA, 2006 Genetic modulation of hormone levels and life span in hybrids between laboratory and wild-derived mice. J Gerontol A Biol Sci Med Sci 61: 1019-1029.

33. Bokov AF, Richardson AG, Chen X, Han ES 2007 Catalase activity up-regulates pro-apoptotic pathways in hepatocytes. In: American Aging Association 36th Annual Meeting, June 1-4, 2007; San Antonio, Texas.

34. Zhou Y, Xu BC, Maheshwari HG, et al, 1997 A mammalian model for Laron syndrome produced by targeted disruption of the mouse growth hormone receptor/binding protein gene (the Laron mouse). Proc Nat Acad Sci USA 94: 13215-13220.

35. Lupu F, Terwilliger JD, Lee K, Segre GV, Efstratiadis A, 2001 Roles of growth hormone and insulin-like growth factor I in mouse postnatal growth. Dev Biol 229: 141162 .

36. Chandrashekar V, Bartke A, Coschigano KT, Kopchick JJ, 1999 Pituitary and testicular function in growth hormone receptor gene knockout mice. Endocrinology 140: 1082-1088.

37. Danilovich N, Wernsing D, Coschigano KT, Kopchick JJ, Bartke A, 1999 Deficits in female reproductive function in GHR-KO mice; role of IGF1. Endocrinology 140: 2637-2640.

38. Zaczek DJ, Hammond JM, Suen L, et al, 2002 Impact of growth hormone resistance on female reproductive function: New insight from growth hormone receptor knockout mice. Biol Reprod 67: 1115-1124.

39. Keene DF, Suescun MO, Bostwick MG, Chandrashekar V, Bartke A, Kopchick JJ, 2002 Puberty in male growth hormone receptor gene disrupted mice. J Androl 23: 661-668.

40. Dominici FP, Arostegui Diaz G, Bartke A, Kopchick JJ, Turyn D, 2000 Compensatory alterations of insulin signal transduction in liver of growth hormone receptor knockout mice. J Endocrinol 166: 579-590.

41. Berryman DE, List EO, Coschigano KT, Behar K, Kim JK, Kopchick JJ, 2004 Comparing adiposity profiles in three mouse models with altered GH signaling. Growth Horm IGF Res 14: 309-318.

42. Coschigano KT, Clemmons D, Bellush LL, Kopchick JJ, 2000 Assessment of growth parameters and life span of GHR/BP gene-disrupted mice. Endocrinology 141: 2608-2613.

43. Bartke A, Chandrashekar V, Bailey B, Zaczek D, Turyn $\mathrm{D}, 2002$ Consequences of growth hormone $(\mathrm{GH})$ overexpression and GH resistance. Neuropeptides 36: 201-208.

44. Bartke A, Wright JC, Mattison JA, Ingram DK, Miller RA, Roth GS, 2001 Longevity: Extending the life span of long-lived mice. Nature 414: 412.

45. Bonkowski MS, Rocha JS, Masternak MM, Al-Regaiey KA, Bartke A, 2006 Targeted disruption of growth hormone receptor interferes with the beneficial actions of calorie restriction. Proc Nat Acad Sci USA 103: 79017905.

46. Miller RA, Chang Y, Galecki AT, et al, 2002 Gene expression patterns in calorically restricted mice: partial overlap with long-lived mutant mice. Mol Endocrinol 16: $2657-2666$.

47. Tsuchiya T, Dhahbi JM, Cui X, et al, 2004 Additive regulation of hepatic gene expression by dwarfism and caloric restriction. Physiol Genomics 17: 307-315.

48. McCurdy CE, Cartee GD, 2005 Akt 2 is essential for the full effect of calorie restriction on insulin-stimulated glucose 
uptake in skeletal muscle. Diabetes 54: 1349-1356.

49. Al-Regaiey KA, Masternak MM, Bonkowski MS, et al, 2007 Effects of caloric restriction and growth hormone resistance on insulin-related intermediates in the skeletal muscle. J Gerontol A Biol Sci Med Sci 62: 18-26.

50. Hiratani K, Haruta T, Tani A, Kawahara J, Usui I, Kobayashi M, 2005 Roles of mTOR and JNK in serine phosphorylation, translocation, and degradation of IRS-1. Biochem Biophys Res Commun 335: 836-842.

51. Taguchi A, Wartschow LM, White MF, 2007 Brain IRS2 signaling coordinates life span and nutrient homeostasis. Science 317: 369-372.

52. Masternak M, Al-Regaiey K, Bonkowski M, et al, 2004, Divergent effects of caloric restriction on gene expression in normal and long-lived mice. J Gerontol A Biol Sci Med Sci 59: 784-788.
53. Masternak MM, Al-Regaiey KA, Del Rosario Lim MM, et al, 2005 Effects of caloric restriction on insulin pathway gene expression in the skeletal muscle and liver of normal and long-lived GHR-KO mice. Exp Gerontol 40: 679-684.

54. Masternak MM, Al-Regaiey KA, Del Rosario Lim MM, et al, 2005 Effects of caloric restriction and growth hormone resistance on the expression level of peroxisome proliferator-activated receptors superfamily in liver of normal and long-lived growth hormone receptor/binding protein knockout mice. J Gerontol A Biol Sci Med Sci 60: 1394-1398.

55. Masternak MM, Al-Regaiey KA, Del Rosario Lim MM, et al, 2006 Caloric restriction and growth hormone receptor knockout: Effects on expression of genes involved in insulin action in the heart. Exp Gerontol 41: 417-429. 